\title{
ChemComm
}

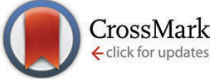

Cite this: Chem. Commun., 2015, 51, 10066

\section{Retraction: N, S co-doped graphene quantum dots from a single source precursor used for photodynamic cancer therapy under two-photon excitation}

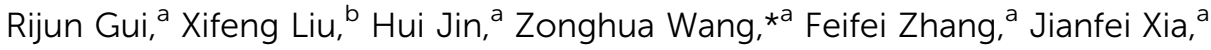 \\ Min Yang, ${ }^{a}$ Sai $\mathrm{Bi}^{\mathrm{a}}$ and Yanzhi Xia ${ }^{a}$
}

DOI: $10.1039 / \mathrm{c5cc} 90244 d$

Retraction of ' $N$, S co-doped graphene quantum dots from a single source precursor used for photodynamic cancer therapy under two-photon excitation' by Rijun Gui et al., Chem. Commun., DOI:

www.rsc.org/chemcomm 10.1039/C4CC09280E.

(1) Xifeng Liu, Zonghua Wang, Feifei Zhang, Jianfei Xia, Min Yang, Sai Bi and Yanzhi Xia wish to resign as co-authors to the above article.

Xifeng Liu has declared that he contributed only to revision of the English language in the manuscript and did not participate in the experimental research reported. Zonghua Wang, Feifei Zhang, Jianfei Xia, Min Yang, Sai Bi and Yanzhi Xia declare that they did not participate in the experimental research that was reported in this article.

The corrected authorship list and affiliations for this paper are as follows:

Rijun Gui, ${ }^{* a}$ and Hui Jin, ${ }^{a}$

${ }^{a}$ The Growing Base for State Key Laboratory, Qingdao University, Qingdao, Shandong 266071, P. R. China. E-mail: guirijun@qdu.edu.cn; Fax: +86 532 85950873; Tel: +86 53285950873

(2) We, the named authors in the corrected authorship list, hereby wholly retract this Chemical Communications article due to data fabrication in the reported characterisation of the polycyclic aromatic hydrocarbon precursor (PTPD) and the realisation subsequent to publication that the reported values for the decomposition rate constant and the ${ }^{1} \mathrm{O}_{2}$ quantum yield for the $\mathrm{N}, \mathrm{S}$ co-doped graphene quantum dots (NS-GQD) are unreliable.

In Part S1 of the ESI the ${ }^{1} \mathrm{H}$-NMR data, ${ }^{13} \mathrm{C}$-NMR data, MALDI-TOF $m / z$ data, and GPC data are copied from the data reported in 'Copolythiophene-Derived Colorimetric and Fluorometric Sensor for Visually Supersensitive Determination of Lipopolysaccharide', J. Am. Chem. Soc., 2012, 134, 6685-6694 for N,N-dimethyl- $N^{\prime}$-(4-(thiophen-3-yl)benzyl)dodecan-1-aminium bromide (compound 4) and the corresponding homopolythiophene PT2. The ${ }^{1} \mathrm{H}-\mathrm{NMR}$ spectrum in Fig. S1b is copied from the ${ }^{1} \mathrm{H}-\mathrm{NMR}$ spectrum of compound 4 reported in Section 1.4 of the ESI of J. Am. Chem. Soc., 2012, 134, 6685-6694.

We have been unable to reproduce the reported decomposition rate constant for ADPA with NS-GQD in subsequent experiments and therefore the reported rate constant cannot be relied upon. This was used in the calculation of the reported ${ }^{1} \mathrm{O}_{2}$ quantum yield. Further to this we have discovered that the equation used to calculate the ${ }^{1} \mathrm{O}_{2}$ quantum yield was incorrect and not the equation reported in Part $\mathrm{S} 3$ of the ESI. Therefore the reported ${ }^{1} \mathrm{O}_{2}$ quantum yield of 1.26 used to support the conclusions of this paper is incorrect and the conclusions cannot be relied upon.

Signed: Rijun Gui and Hui Jin, 15th April 2015

Retraction endorsed by May Copsey, Executive Editor, Chemical Communications.

\footnotetext{
${ }^{a}$ Collaborative Innovation Center for Marine Biomass Fiber, Materials and Textiles of Shandong Province, Shandong Sino-Japanese Center for Collaborative Research of Carbon Nanomaterials, Laboratory of Fiber Materials and Modern Textiles, the Growing Base for State Key Laboratory, College of Chemical Science and Engineering, Qingdao University, Qingdao, Shandong 266071, P. R. China. E-mail: wangzonghua@qdu.edu.cn; Fax: +86 532 85950873; Tel: +8653285950873

${ }^{b}$ Department of Orthopedic Surgery, Mayo Clinic, Rochester, MN 55905, USA
} 